\title{
Kinetic Studies of the Oxidative Assimilation of Acetate by a Non-photosynthetic Strain of Euglena gracilis
}

\author{
By G. MARZULLO AND W. F. DANFORTH \\ Department of Biology, Illinois Institute of Technology, Chicago 16, Illinois, U.S.A.
}

(Received 24 April 1963)

\begin{abstract}
SUMMARY
Kinetic studies of the effects of unlabelled acetate on ${ }^{14} \mathrm{CO}_{2}$ production by a non-photosynthetic strain of ${ }^{14} \mathrm{C}$-labelled Euglena indicate the existence of a 'labile reserve', containing 1.5-2.0 $\mu$ g.-atoms $\mathrm{C} / \mathrm{million}$ organisms, which is an early product of acetate assimilation and a substrate of endogenous metabolism. When this Euglena assimilated radioactive acetate, radioactivity in an ethanol-soluble form (Soluble I) increased rapidly at first, then levelled off at a value corresponding to about $0 \cdot 2 \mu \mathrm{g}$.-atom $\mathrm{C} / \mathrm{million}$ organisms. Ethanol-insoluble radioactivity appeared slowly at first, then at an increasing rate which became linear at about 30-60 min. after addition of acetate. Kinetic analyses suggest that Soluble $I$ is the precursor of the insoluble material. A second ethanol-soluble component began to become labelled some time after the first hour, and reached maximum radioactivity, corresponding to about $0.5 \mu \mathrm{g}$-atom $\mathrm{C} /$ million organisms, $2-3 \mathrm{hr}$ after addition of tracer acetate. The chemical composition of the ethanol-soluble and ethanol-insoluble components and their relationship to the 'labile reserve' are discussed.
\end{abstract}

\section{INTRODUCTION}

During oxidative assimilation of acetate by a non-photosynthetic strain of Euglena gracilis var. bacillaris, $42 \%$ of the acetate-carbon appeared as $\mathrm{CO}_{2}$, while the remaining $\mathbf{5 8} \%$ was assimilated. Carbon and oxygen balance studies indicated

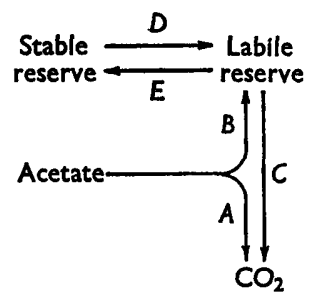

Fig. 1. Diagram of the postulated role of the 'labile reserve' in acetate metabolism of Euglena. $A$ is the oxidation pathway for acetate; $B$ is the pathway of acetate assimilation; $C$ is the pathway of $\mathrm{CO}_{2}$ production from intracellular reserves; $D$ and $E$ are exchange reactions between the 'labile reserve' and other reserve substances. (After Danforth \& Wilson, 1961.)

that the major assimilatory products have the empirical composition of carbohydrate (Wilson \& Danforth, 1958; Danforth, 1961). Oxidation of cellular reserves continues during the oxidative assimilation of acetate (Wilson \& Danforth, 1958). Tracer studies with radioactive carbon led Danforth \& Wilson (1961) to suggest 
that the most recently assimilated acetate-carbon forms a 'labile reserve', which is also the immediate substrate for the reserve oxidation process. This 'labile reserve' was believed to undergo a slow exchange with a much larger 'stable reserve' (Fig. 1). The data presented at that time were not adequate to estimate the capacity of the 'labile reserve', nor to indicate whether the 'labile reserves' consisted of a homogeneous pool or of several compartments. The present study is directed toward further investigation of these problems.

\section{METHODS}

General methods. The non-photosynthetic strain of Euglena gracilis var. bacillaris used, the general methods of growth and handling of the euglenas, and the respirometric tracer techniques were as described in previous publications (Danforth, 1953, 1961; Danforth \& Wilson, 1957, 1961; Wilson \& Danforth, 1958).

Buetow (1961) showed that the respiratory rate of Euglena decreases with increasing centrifugation during harvesting. The harvesting procedure used routinely in our laboratory regularly yields euglenas with respiratory rates of 20 and $30 \mu \mathrm{l} . \mathrm{O}_{2} /$ million euglenas $/ \mathrm{hr}$., well below the 50-60 $\mu \mathrm{l} . / \mathrm{million}$ euglenas $/ \mathrm{hr}$ found by Buetow after minimal centrifugation. We have seen no indication that this decreased metabolic rate alters the patterns of carbon distribution which are the subject of the present work, but this problem clearly deserves further study.

The 'labile reserve'. Labelled euglenas were grown in 11 . Erlenmeyer flasks containing $500 \mathrm{ml}$. of medium composed of (w/v): Bacto-Tryptone (Difco), $0 \cdot 3 \%$; sodium acetate $\mathbf{3 H}_{2} \mathrm{O}, \mathbf{0 \cdot 3} \%$. The acetate was uniformly labelled with $80 \mu \mathrm{C}$. ${ }^{14} \mathrm{C}$ per flask. Cultures were inoculated with $5 \mathrm{ml}$. of a heavy suspension of euglenas, and incubated for 5 days at $26^{\circ}$.

Experimental euglenas were incubated in special $50 \mathrm{ml}$. Erlenmeyer flasks (equipped with centre wells) containing $13 \mathrm{ml}$. of 20 mM-sodium acetate (non-

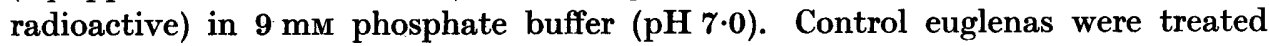
similarly, except that acetate was omitted from the medium. The centre wells in the flasks contained $1.0 \mathrm{ml} .5 \mathrm{M}-\mathrm{NaOH}$ plus filter paper. Incubation was at about $26^{\circ}$, with shaking at 120 cycles $/ \mathrm{min}$. Samples were removed at intervals, centrifuged, washed, and resuspended in fresh medium. Samples $(1.5 \mathrm{ml}$.) of these fresh suspensions were transferred to Warburg flasks whose centre wells contained $0.1 \mathrm{ml} .5 \mathrm{M}-\mathrm{NaOH}$ (without filter paper), and whose side arms contained $0.5 \mathrm{ml}$. $\mathrm{M}-\mathrm{H}_{2} \mathrm{SO}_{4}$. After incubation with shaking for the desired intervals, the side arms were tipped, thus killing the euglenas and acidifying the medium. The flasks were shaken for a further $2 \mathrm{hr}$ to ensure complete transfer of $\mathrm{CO}_{2}$ to the centre wells. The centre-well contents were withdrawn, and the centre wells rinsed twice, first with $0.3 \mathrm{ml}$., then with $0.4 \mathrm{ml}$. distilled water. The centre-well contents and rinses were pooled, transferred to planchets, dried under an infrared lamp, and counted for radioactivity. A drop of ethanol was added to each planchet before drying to facilitate uniform spreading of the sample. Similar samples were transferred at the beginning of the experiment to Warburg flasks for measurement of respiration. Filter paper was added to the centre wells of these flasks, $\mathrm{H}_{2} \mathrm{SO}_{4}$ being omitted from the side arms. Oxygen consumption of experimental and control euglena suspensions was followed for the entire experimental period. 
Kinetics of acetate assimilation. Euglenas were grown at about $\mathbf{2 6}^{\circ}$ in a medium consisting of $(\mathrm{w} / \mathrm{v})$ Bacto Tryptone, $0.3 \%$; sodium acetate $\mathbf{3 H}_{2} \mathrm{O}, 0.5 \%$. The euglenas were incubated at $26^{\circ}$, with shaking at 120 cycles $/ \mathrm{min}$., in stoppered 50 or $120 \mathrm{ml}$. Erlenmeyer flasks equipped with centre wells. The main compartments of the flasks contained: acetate- ${ }^{14} \mathrm{C}, 10 \mathrm{mM}$; phosphate buffer ( $\mathrm{pH} \mathrm{7 \cdot 0}$ ), $10 \mathrm{mM} ; 1.5-4.8 \mathrm{million}$ euglenas $/ \mathrm{ml}$. Uniformly labelled acetate was used in some experiments, methyl-labelled acetate in others. Centre wells contained $0.3 \mathrm{ml}$. $5 \mathrm{M}-\mathrm{NaOH}$ and a square of filter paper. At intervals, $0.5 \mathrm{ml}$. samples were withdrawn from the main compartments and pipetted into centrifuge tubes containing $0.8 \mathrm{ml} .95 \%(\mathrm{v} / \mathrm{v})$ ethanol in water, giving a final ethanol concentration of $60 \%$ $(\mathrm{v} / \mathrm{v})$. The samples were heated to $65^{\circ}$ for $30 \mathrm{~min}$., then centrifuged, and the supernatant fluid decanted into a second tube. The precipitate was washed by recentrifugation from the same volume of $60 \%$ ethanol, and resuspended in $1.3 \mathrm{ml}$. $60 \%$ ethanol. Samples of the original supernatant fluid and of the resuspended precipitate were transferred to planchets and dried under an infrared lamp. Before counting, the dry samples were flooded twice with $0.5 \mathrm{ml}$. glacial acetic acid, drying each time, to drive off residual acetate. (In the initial experiments, samples of the ethanol wash were prepared in similar fashion. Since no radioactivity was found in these washes, they were discarded in later experiments.) An initial sample was prepared at zero time by pipetting $0.5 \mathrm{ml}$. of the reaction mixture into $0.8 \mathrm{ml}$. $80 \%(\mathrm{v} / \mathrm{v})$ ethanol containing $0 \cdot 1 \mathrm{M}-\mathrm{NaOH}$; this sample was dried and counted directly to determine the specific activity of the acetate initially present.

For measurements of respiration, two Warburg flasks were prepared in each experiment, one containing the same reaction mixture as in the experimental flask (except that the acetate was non-radioactive), and one in which acetate was omitted.

Assimilation of acetate carbon into ethanol-soluble and ethanol-insoluble fractions was determined by counting the radioactivity of the appropriate planchets. Total acetate consumption could be estimated by using the stoichiometric carbon-oxygen balance equations of Wilson \& Danforth (1958; Danforth, 1961), either from the respiration or independently from the amount of acetate-carbon assimilated. In some experiments, utilization was determined directly by counting the radioactivity remaining in the extracellular medium. All three methods gave equivalent results, within the expected precision of the methods; estimation from respiration was most convenient and was generally used.

\section{RESULTS}

\section{Capacity of the 'labile reserve'}

If it be assumed that the 'labile reserve' consists of a homogeneous pool of fixed capacity, $V$, it can be shown (see Theoretical Appendix) that during the oxidation of non-labelled acetate by Euglena uniformly labelled with ${ }^{14} \mathrm{C}$ :

$$
\ln \frac{(C+E) R-D}{B}=\frac{-(C+E) t}{V},
$$

where $\boldsymbol{R}$ is the specific activity of the carbon in the 'labile reserve', $t$ is the time after addition of unlabelled acetate, and $B, C, D$ and $E$ are the rates of the corresponding 
reactions shown in Fig. 1. These rates can be evaluated from the experimental data (see Theoretical Appendix). Plotting $\ln [\{(C+E) R-D\} / B]$ against $(C+E) t$ should give a straight line through the origin, with slope equal to $-1 / V$.

Figures 2 and 3 show an example of the experimental evaluation of $V$ by this method; Table 1 presents the numerical results of this experiment. For this experiment, the line of best fit (by eye) yielded a value for $V$ of $1.70 \mu \mathrm{g}$.-atoms $\mathrm{C} /$ million

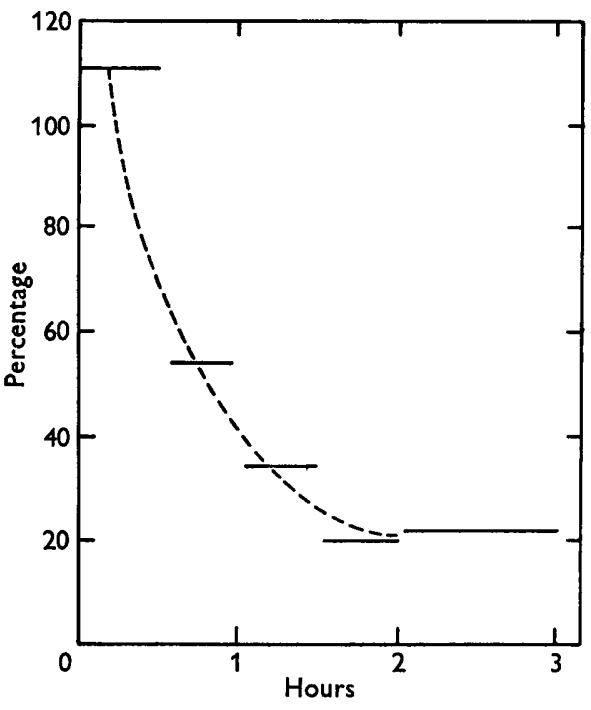

Fig. 2

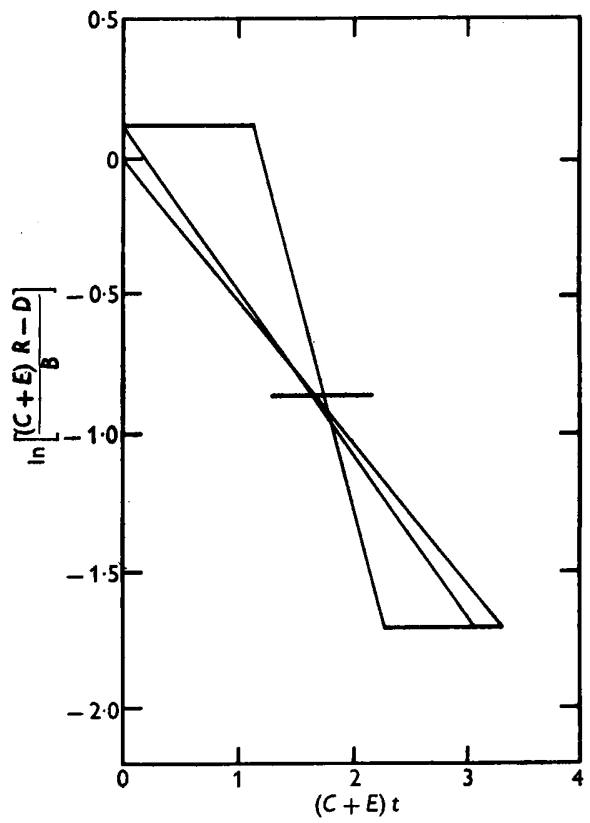

Fig. 3

Fig. 2. Effect of unlabelled acetate on the production of ${ }^{14} \mathrm{CO}_{2}$ by ${ }^{14} \mathrm{C}$-labelled euglenas. Rate of ${ }^{14} \mathrm{CO}_{2}$ production is expressed as percentage of the rate in the absence of acetate. Each horizontal line is the average rate over a single period of measurement.

Fig. 3. Same data as in Fig. 2, plotted to provide an estimate of the capacity of the 'labile reserve'.

Table 1. Numerical values of constants in equation (5), and the resulting estimates of $\mathrm{V}$, for the experiment shown in Figs. 2 and 3

Constants $\boldsymbol{A}-\boldsymbol{E}$ are the rates of corresponding reactions in Fig. 1, estimated as described in the Theoretical Appendix. $V$ is the capacity of the 'labile reserve', estimated graphically from Fig. 3.

Pathway or fraction

$$
\begin{aligned}
& \boldsymbol{A} \\
& \boldsymbol{B} \\
& \boldsymbol{C} \\
& \boldsymbol{D} \\
& \boldsymbol{E}
\end{aligned}
$$

$V$ (best)

$V$ (maximum)

$V$ (minimum)
Value ( $\mu$ g.-atoms $\mathrm{C} / \mathbf{1 0}^{6}$ euglenas/hr.)

$\mathbf{1} \cdot \mathbf{2 8}$

$1 \cdot 76$

$0 \cdot 18$

$0 \cdot 44$

1.01

( $\mu$ g.-atoms $\mathrm{C} / \mathbf{1 0}^{6}$ euglenas)

$1 \cdot 70$

1.95

0.58 
euglenas. A second experiment gave an estimated $V$ of $1.55 \mu$ g.-atoms $\mathrm{C} / \mathrm{million}$ euglenas by the same procedure. Extreme estimates of $V$ from the same two experiments ranged from 0.58 to $1.95 \mu \mathrm{g}$.-atoms C/million euglenas. Despite the uncertainties of the calculation, it seems likely that the actual amount of material making up the 'labile reserve' falls somewhere within this range.

A simpler, partially independent estimate of the capacity of the 'labile reserve' may be obtained directly from the data presented in Fig. 2 and Table 1. The rate of ${ }^{14} \mathrm{CO}_{2}$ production at about $40-50 \mathrm{~min}$. is halfway between the initial and final rates. It may be assumed that this degree of 'dilution' occurs when the amount of unlabelled $\mathrm{C}$ assimilated is slightly more than one-half the capacity of the labile reserve. From Table 1, the assimilation at this time is about 1.0-1.3 $\mu$ g.-atoms $\mathrm{C} /$ million euglenas, again suggesting that $V$ is in the neighbourhood of $2 \mu$ g.-atoms $\mathrm{C} /$ million euglenas.

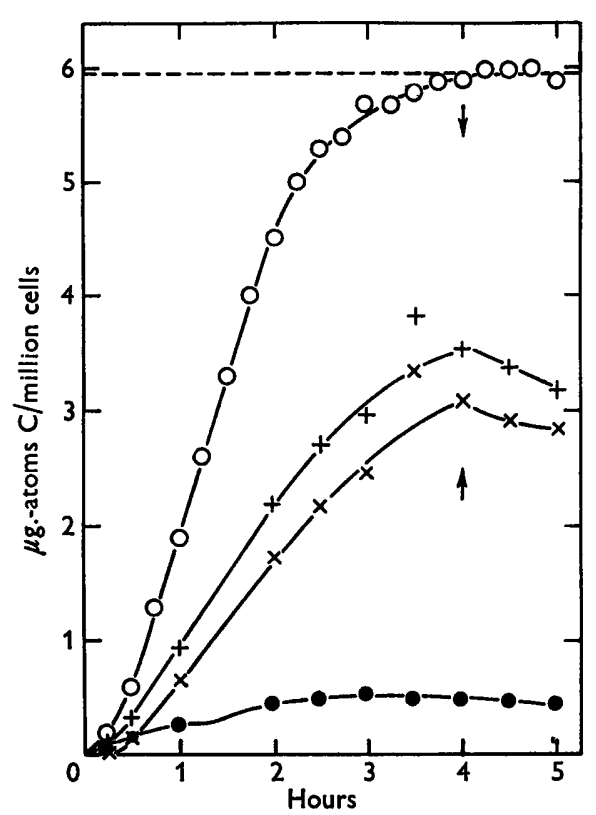

Fig. 4

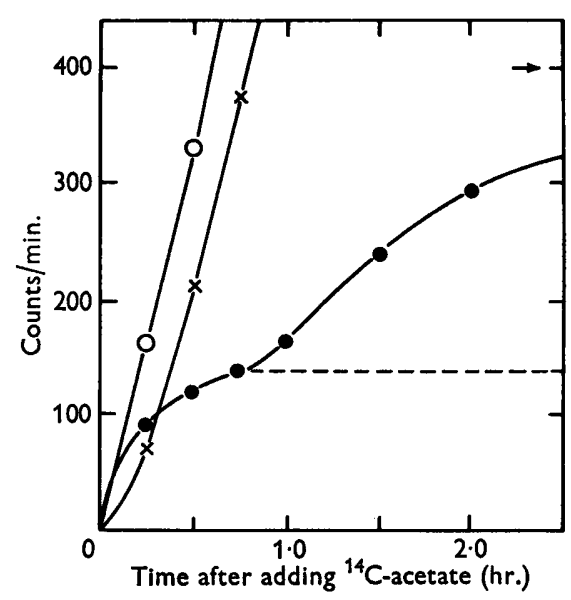

Fig. 5

Fig. 4. Time course of metabolism of ${ }^{14} \mathrm{C}$-acetate. $O$, Total acetate consumption; + , total assimilation; $x$, assimilation into ethanol-insoluble material; $O$, assimilation into ethanol-soluble material. Arrows indicate the time of exhaustion of acetate; dotted line indicates the amount of acetate initially present.

Fig. 5. Early stages of assimilation of ${ }^{14} \mathrm{C}$-acetate. $O$, Total assimilation; $x$, assimilation into ethanol-insoluble material; $\bigcirc$, assimilation into ethanol-soluble material. Dotted line shows estimated maximum ${ }^{14} \mathrm{C}$ content of Soluble I. Arrow shows ultimate maximum value reached by ethanol-soluble fraction.

\section{Kinetics of assimilation of radioactive acetate}

Study of the assimilation of radioactive acetate by non-radioactive euglenas provides an independent means for studying the metabolic processes concerned in the supposed 'labile reserve'; Fig. 4 shows the results of a typical experiment. 
As concluded from previous experiments (Wilson \& Danforth, 1958; Danforth, 1961) acetate consumption (after a very brief lag) occurred at a constant rate until the acetate was nearly exhausted from the medium, and the fraction of this acetate assimilated was essentially constant during the entire course of the experiment. Assimilatory products soluble in $60 \%$ ethanol accumulated rapidly at first, but soon reached a relatively constant 'plateau' of about $0.5 \mu$ g.-atom C/million euglenas. Ethanol-insoluble products, on the other hand, appeared slowly at first, then at an increasing rate, and finally increased linearly until the acetate was exhausted.

The general appearance of the curves in Fig. 4 suggests that the ethanol-soluble products were precursors of the ethanol-insoluble products. Closer examination, however, suggests that the situation was more complex. Fig. 5 shows the early stages of an experiment similar to that shown in Fig. 4, plotted on a larger scale.

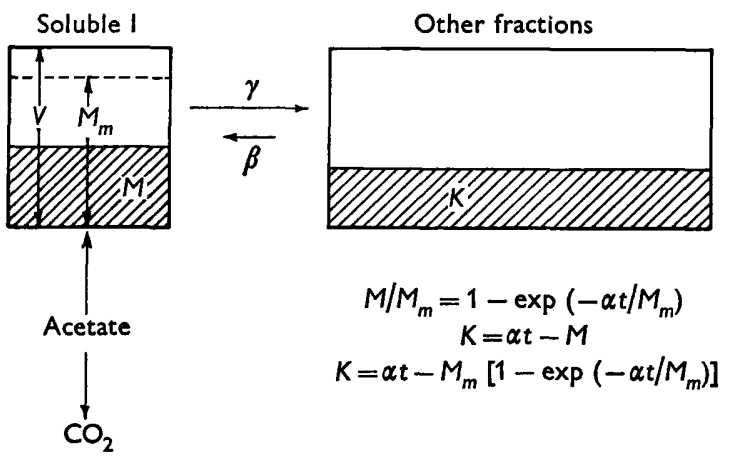

Fig. 6. Model used in kinetic analysis of acetate assimilation. See text for definitions of symbols.

In this case, it is apparent that the ethanol-soluble products accumulate in two stages, one beginning immediately and reaching a plateau after about the first hour, the second beginning after the first hour, and reaching a plateau only after 2 or more hours. A similar two-stage accumulation of ethanol-soluble products was observed in every experiment suitable for such analysis.

Of these two subdivisions of the soluble products, only the first to appear (hereafter designated Soluble I) has the characteristics expected of a precursor of the insoluble material. Examination of Fig. 5 shows that the rate of accumulation of insoluble radioactivity is roughly proportional to the radioactivity of Soluble I, and reaches a constant maximum at about the time Soluble I reaches a plateau, as would be expected if Soluble I were the immediate precursor of the insoluble material. The second stage of accumulation of soluble material ('Soluble II'), on the other hand, begins to appear only after the insoluble radioactivity is accumulating at maximum rate, a relationship incompatible with the hypothesis that Soluble II is a precursor of the insoluble products. Instead, the time relations suggest that Soluble II is derived either from Soluble I or, more probably, from the insoluble material.

The assumption that Soluble $I$ is the precursor of both the insoluble products 
and of Soluble II can be tested more critically by making use of the model shown in Fig. 6, and the equations derived from this model :

$$
\begin{gathered}
M / M_{m}=1-\exp \left(-\alpha t / M_{m}\right), \\
K=\alpha t-M,
\end{gathered}
$$

where $M$ is the amount of ${ }^{14} \mathrm{C}$ in Soluble $\mathrm{I}, K$ is the sum of the amounts of ${ }^{14} \mathrm{C}$ in Soluble II and the insoluble fraction, $t$ is time, and $\alpha$ and $M_{m}$ are constants subject to experimental evaluation (see Theoretical Appendix).

Figure 7 compares the experimental course of appearance of radioactivity with the expected results calculated from the above equations; the experiment is that shown in Fig. 5. The agreement between theory and experiment is fairly good,

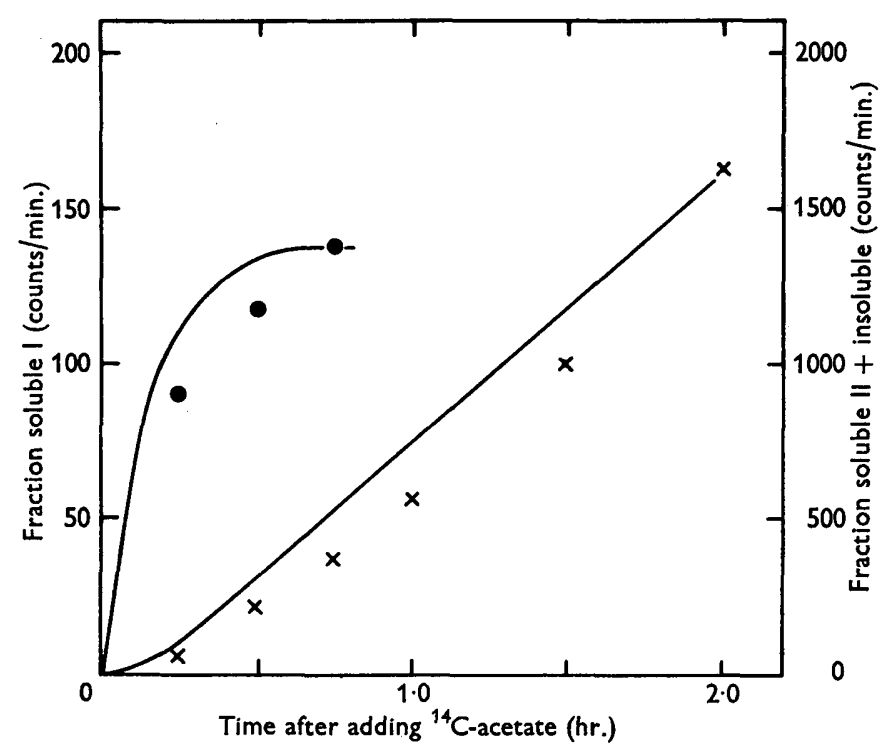

Fig. 7. Comparison of the actual time course of acetate assimilation with that predicted by the model shown in Fig. 6. and left ordinate, Soluble $I ; \times$ and right ordinate, Soluble II plus insoluble. Points are experimental data, lines are calculated.

except that the experimental values consistently lag behind the theoretical by about 10-15 $\mathrm{min}$. This systematic deviation can probably be accounted for by the fact that $\alpha$, the rate of total assimilation, was assumed to be constant over the entire time period. In actual fact, the rate of acetate consumption during the first few minutes of such experiments was consistently found to be slower than the later steady-state rate (see Fig. 4). The amount of this delay, 10-20 min., is of the right order to explain the lag in the experimental values. It would seem, therefore, that the model shown in Fig. 6, in which Soluble $I$ is considered to be a single homogeneous pool, the first product of acetate assimilation, and the precursor of all other assimilation products, gives a reasonable approximation of the actual events. 


\section{Amounts of the ethanol-soluble products}

Table 2 presents the maximum (plateau) values of ${ }^{14} \mathrm{C}$ found in the two subdivisions of the ethanol-soluble fraction in several experiments of the type just described. The total carbon content of these pools may be somewhat greater than these values because of the possibility that back reactions occur, which feed a certain amount of non-radioactive carbon into the pools (e.g. reaction $\beta$ in Fig. 6). The magnitude of such possible back reactions is unknown, but is probably not large for intermediates on the direct pathway of acetate assimilation. Any extensive recycling of carbon between intermediates and products of assimilation would be expected to produce randomization between the methyl and carboxyl carbons of acetate; in fact, the fates of the two carbons are markedly different (Danforth, 1961). In Polytomella coeca, whose acetate metabolism resembles that of Euglena in many respects (Danforth, 1961), this lack of randomization is apparent even in long-term growth experiments (Bevington, Bourne \& Turton, 1953; Barker \& Bourne, 1955). Similarly, recycling should produce apparent changes in oxidationassimilation ratios, and in the relative fates of methyl and carboxyl carbons, during the course of acetate utilization; no such changes have been detected (Danforth, 1961). It seems probable, therefore, that acetate assimilation is largely a one-way process, and that back reactions do not result in any gross underestimate of the pool sizes presented in Table 2.

Table 2. Maximum values of ${ }^{14} \mathrm{C}$ found in the ethanol-soluble fractions

Data from four experiments of the type shown in Fig. 4 were interpreted graphically by the method shown in Fig. 5 .

Ethanol-soluble fraction

\begin{tabular}{|c|c|c|c|}
\hline \multirow{2}{*}{ Expt. no. } & Soluble I & Soluble II & Total soluble \\
\hline & \multicolumn{3}{|c|}{ Maximum values of ${ }^{14} \mathrm{C}$ ( $\mu$ g.-atom $\mathrm{C} / 10^{6}$ euglenas) } \\
\hline M1 & $0 \cdot 22$ & 0.54 & 0.76 \\
\hline M3 & $0 \cdot 17$ & $\mathbf{0} \cdot \mathbf{3 2}$ & $0 \cdot 49$ \\
\hline M4 & 0.23 & $\mathbf{0} \cdot \mathbf{3 0}$ & 0.53 \\
\hline M6b & $0 \cdot 14$ & $0 \cdot 13$ & $0 \cdot 27$ \\
\hline Mean & $0 \cdot 19$ & $\mathbf{0 . 3 2}$ & 0.51 \\
\hline
\end{tabular}

Nature of the assimilation products. It has been shown (Marzullo \& Danforth, 1964) that nearly $90 \%$ of the acetate carbon found in ethanol-insoluble products can be recovered in the glucose units of the reserve polysaccharide, paramylon, which occurs as granular inclusions within the Euglena organisms. The nature of the ethanol-soluble subfractions is relatively unknown. If the scheme shown in Fig. 6 be correct, Soluble I should consist largely of intermediates in the conversion of acetate to carbohydrates, presumably Krebs cycle acids, the phosphorylated intermediates of glucose metabolism and oligosaccharides. In partial support of this theory, Lynch \& Calvin $(1952,1953)$ found that, of the acetate methyl-carbon incorporated into ethanol-soluble materials during incubation for $12 \mathrm{~min}$. in the dark, nearly $50 \%$ was in the form of phosphorylated intermediates, $4 \%$ in the form of 'dextrins', and about $25 \%$ in the form of amino acids related to the Krebs cycle. 
Even less is known about the composition of Soluble II; investigations of the components of both soluble subfractions are under way at present.

Relation of the 'labile reserves' to ethanol fractions. Of the three carbon pools inferred from the kinetics of assimilation of radioactive acetate, only Soluble I shows kinetic characteristics similar to those postulated for the 'labile reserve', rising to a maximum during the first hour or so after addition of acetate, and remaining at a constant level thereafter. There is, however, a great discrepancy between the estimated size of Soluble I and that of the 'labile reserve'. The capacity of the 'labile reserve' was estimated to be in the neighbourhood of 1.5-2 $\mu \mathrm{g}$.atoms $\mathrm{C} /$ million euglenas, while that of Soluble $\mathrm{I}$ is only about one-seventh as great, about 0.2 $\mu \mathrm{g}$--atom $\mathrm{C} /$ million euglenas. Even allowing for the considerable uncertainties involved in both estimates, this quantitative difference seems to preclude the possibility that the 'labile reserve' is identical with Soluble I. Indeed, the 'labile reserve' seems to be about three times as large as Soluble I and Soluble II together, which strongly suggests that the 'labile reserve' includes insoluble as well as soluble components. Since the insoluble materials are so largely paramylon, this probably indicates that paramylon contributes to the 'labile reserve'.

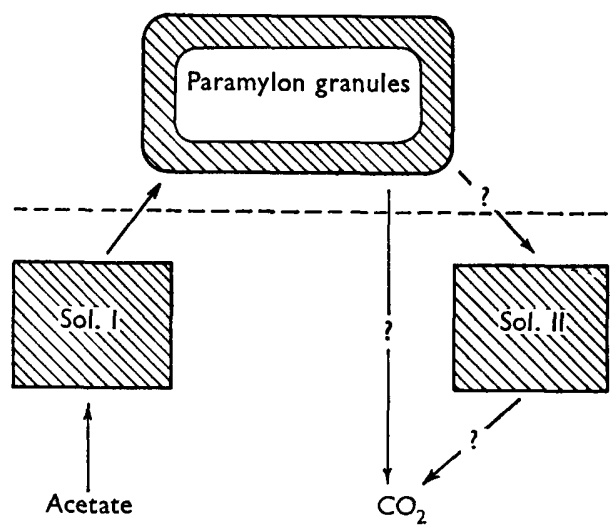

Fig. 8. Hypothetical explanation of the relationships among the products of acetate assimilation. Cross-hatched portions are believed to constitute the 'labile reserve'. Materials above the dotted line are considered to be ethanol-insoluble; materials below the line, ethanol-soluble.

To account for these relationships, it is suggested that the 'labile reserve' consists in part of the surface layers of some or all of the paramylon granules. It would be expected that newly synthesized paramylon would be deposited on the surfaces of the granules, and that these surface layers would be the first portion of the granules to be attacked by degradative enzymes. Thus the finding that newly assimilated carbon is the first to be oxidized by the endogenous pathway can be accounted for. It was this finding which led originally to the concept of a 'labile reserve'. The intermediates in the conversion of acetate to paramylon (presumably included in Soluble I) would also contribute to the 'labile reserve' (Fig. 8). The lag which is sometimes apparent in the dilution of endogenous $\mathrm{CO}_{2}$ production by exogenous acetate (Fig. 2) suggests that the 'labile reserve' contains more than one component. The place of Soluble II in this scheme is not clear. Soluble II might 
consist of intermediates in the oxidation of acetate via the endogenous pathway, or of by-products derived from paramylon by side reactions. The very late appearance of radioactivity in Soluble II seems to favour the latter possibility.

\section{THEORETICAL APPENDIX}

Kinetics of the 'labile reserve'. Assume the model system presented in Fig. 1, and further assume that the 'labile reserve' is a homogeneously mixed pool of fixed capacity, $V$. When non-radioactive acetate is supplied to fully labelled euglenas, the amount of ${ }^{14} \mathrm{C}$ in the 'labile reserve' will decrease according to the relationship

$$
\frac{\mathrm{d} X}{\mathrm{~d} t}=D-\frac{X}{V}(C+E),
$$

where $X=$ amount of ${ }^{14} \mathrm{C}$ in the 'labile reserve', and $C, D$, and $E$ the rates of the corresponding reactions in Fig. 1. Letting $X / V=R=$ specific activity of carbon in the 'labile reserve',

$$
\frac{\mathrm{d} R}{\mathrm{~d} t}=\frac{D-(X / V)(C+E)}{V} .
$$

Integrating equation (2) and evaluating the constant of integration for the values $(t=0, R=1 \cdot 0)$, gives

$$
-\ln [(C+E) R-D]=\frac{(C+E)}{V}-\ln (C+E-D) .
$$

But, if $V$ is constant,

$$
B+D=C+E, \quad \text { or } \quad C+E-D=B .
$$

Substituting from (4) into (3),

$$
\begin{gathered}
-\ln [(C+E) R-D]=\frac{(C+E) t}{V}-\ln B, \\
\ln \left[\frac{(C+E) R-D}{B}\right]=-\frac{(C+E) t}{V} .
\end{gathered}
$$

From Fig. 1 it may be seen that $B$ is the rate of conversion of acetate carbon to reserves, which may be determined from the respiration by using the carbon:oxygen balance equations of Wilson \& Danforth (1958; Danforth, 1961). C, the rate of $\mathrm{CO}_{2}$-production from reserves, is equal to the endogenous oxygen consumption, since the endogenous respiratory quotient is $1 \cdot 0$ (Danforth \& Wilson, 1961).

Since the 'labile reserve' is considered to be the direct precursor of the endogenous $\mathrm{CO}_{2}$, the specific activity $(R)$, of the 'labile reserve' (relative to that of control cells without acetate) equals

$$
\frac{\text { Rate of }{ }^{14} \mathrm{CO}_{2} \text { production by experimental euglenas }}{\text { Rate of }{ }^{14} \mathrm{CO}_{2} \text { production by control euglenas }}
$$

and is experimentally determined (Fig. 2).

Solving equation (5) for $\boldsymbol{R}$ gives

$$
R=\frac{B \exp [-(C+E) t / V]+D}{C+E},
$$


or, substituting $B+D=C+E$ from equation (4),

$$
R=\frac{B \exp [-(C+E) t / V]+D}{B+D}
$$

As $t$ increases, the exponential term in equations (6) and (6a) approaches zero, and $\boldsymbol{R}$ approaches a steady-state value

$$
\boldsymbol{R}(\text { steady state })=\frac{D}{B+D}
$$

Since $B$ is known, $D$ may be calculated from the steady-state value of $R$. $E$ may then be estimated from equation (4), using values of $B, C$ and $D$ derived as described above.

Kinetics of the ethanol fractions. Assume the relationship indicated in Fig. 6 between Soluble I and the other assimilatory products, and further assume that Soluble $I$ is a homogeneously mixed pool of fixed capacity $W$. If uniformly labelled acetate $-{ }^{14} \mathrm{C}$ is supplied to initially unlabelled euglenas,

$$
\frac{\mathrm{d}(M / W)}{\mathrm{d} t}=\frac{\alpha-\gamma(M / W)}{W},
$$

where $M=$ amount of ${ }^{14} \mathrm{C}$ in Soluble $\mathrm{I}$, and $\alpha$ and $\gamma$ are the rates of the corresponding reactions in Fig. 6. Integrating, and evaluating the constant of integration for $(t=0, M=0)$ gives

$$
\frac{M}{W}=\frac{\alpha}{\gamma}[1-\exp (-\gamma t / W)]
$$

Neither $\gamma$ or $W$ are subject to experimental evaluation. But, as $t$ becomes large, $\boldsymbol{M}$ approaches a constant maximum value $\boldsymbol{M}_{m}$, and $\exp (-\gamma t / W)$ approaches zero, giving

$$
\frac{M_{m}}{W}=\frac{\alpha}{\gamma}, \quad W=\frac{\gamma}{\alpha} M_{m}
$$

Substituting this expression for $W$ in equation (9) gives

$$
\frac{M}{M_{m}}=1-\exp \left(-\alpha t / M_{m}\right)
$$

in which all quantities can be experimentally evaluated.

Also, from the initial assumptions,

$$
\frac{\mathrm{d} K}{\mathrm{~d} t}=\frac{\gamma M}{W}=\frac{\alpha M}{M_{m}}
$$

where $K$ is the amount of label in all fractions other than Soluble I. Integrating (12), and evaluating the constant of integration for $(t=0, K=0)$, gives

$$
K=\alpha t-M_{m}\left[1-\exp \left(-\alpha t / M_{m}\right)\right]=\alpha t-M \text {. }
$$

The total assimilation after $2 \mathrm{hr}$. was used to evaluate $\alpha$, assuming the assimilatory rate to be constant over this period. $M_{m}$ was estimated graphically as illustrated in Fig. 5, while $K, M$, and $t$ are derived directly from the experimental data. 
This investigation was supported in part by a Public Health Service Research Grant RG-7918 from the Division of General Medical Sciences, U.S. Public Health Service, and includes portions of a thesis presented by G. Marzullo in partial fulfilment of the requirements for the degree of Master of Science at Illinois Institute of Technology. Some of the results were presented at the First International Conference on Protozoology, Prague, 1961, and will appear in abbreviated form in the Proceedings of that Conference.

\section{REFERENCES}

Barker, S. A. \& Bourne, E. J. (1955). In Biochemistry and Physiology of Protozoa. Ed. by S. H. Hutner and A. Lwoff, vol. II, p. 45. New York: Academic Press Inc.

Bevington, J. C., Bourne, E. J. \& Turton, C. N. (1953). Chemical degradation of ${ }^{14} \mathrm{C}$-glucose and its application to ${ }^{14} \mathrm{C}$-starch from Polytomella coeca. Chem. $\&$ Ind. p. 1390.

BUETOw, D. E. (1961). Variation of the respiration of protozoan cells with length of centrifuging. Analyt. Biochem. 2, 242.

Danforth, W. (1953). Oxidative metabolism of Euglena. Arch. Biochem. 46, 164.

Danforth, W. F. (1961). Oxidative assimilation of acetate by Euglena. Carbon balance and effects of ethanol. J. Protozool. 8, 152.

Danforth, W. F. \& Wilson, B. W. (1957). Adaptive changes in the acetate metabolism of Euglena. J. Protozool. 4, 52.

Danforth, W. F. \& Wilson, B. W. (1961). The endogenous metabolism of Euglena gracilis. J. gen. Microbiol. 24, 95.

LyNCh, V. H. \& Calvin, M. (1952). Carbon dioxide fixation by microorganisms. J. Bact. 63, 525.

Lynch, V. H. \& Calvin, M. (1953). $\mathrm{CO}_{2}$ fixation by Euglena. Ann. N.Y. Acad. Sci. 56, 890.

Marzullo, G. \& Danforth, W. F. (1964). Composition of ethanol-insoluble assimilatory products of oxidative assimilation of acetate by Euglena gracilis. J. gen. Microbiol. 34, 21.

Wilson, B. W. \& Danforth, W. F. (1958). The extent of acetate and ethanol oxidation by Euglena gracilis. J. gen. Microbiol. 18, 535. 